\title{
THERE IS JUST ONE RATIONAL CONE-LENGTH
}

\author{
OCTAVIAN CORNEA
}

\begin{abstract}
We show that the homotopic nilpotency of the algebra of piecewise polynomial forms on a simply-connected, finite type, CW-complex coincides with the strong L.S. category of the rationalization of that space. This is used to prove that, in the rational, simply-connected context all reasonable notions of cone-length agree. Both these two results are obtained as parts of a more general and functorial picture.
\end{abstract}

\section{INTRODUCTION}

Let $\mathscr{S}$ be the pointed category of pointed spaces of the homotopy type of a $\mathrm{CW}$-complex and let $\mathscr{F}=\mathscr{F}_{0} \subset \mathscr{F}_{1} \subset \mathscr{F}_{2} \subset \cdots \subset \mathscr{F}_{n} \subset \cdots$ be a filtered class of objects of $\mathscr{S}$ with the property that each $\mathscr{F}_{i}$ is closed with respect to wedges and suspensions and the zero dimensional sphere is in $\mathscr{F}_{0}$.

Definition 1. For $X \in \mathscr{O} b(\mathscr{S})$ the cone-length of $X$ with respect to $\mathscr{F}$, $\mathrm{Cl}_{\mathscr{F}}(X)$, is the least natural number $n$ for which there are cofibration sequences:

$$
Z_{i} \rightarrow X_{i} \rightarrow X_{i+1}
$$

for $0 \leq i<n$, with $X_{0}=*, X_{n} \simeq X$ and $Z_{i} \in \mathscr{F}_{i}$ (if no such number exists, take $\left.\mathrm{Cl}_{\mathscr{F}}(X)=\infty\right)$.

Remark. Obviously, similar notions may be defined in more general categories where homotopy theory is possible.

Examples. 1. $\mathscr{F}_{i}=\mathscr{O} b(\mathscr{S})$ for all $i$. Then $\mathrm{Cl}_{\mathscr{F}}=$ Cat , the strong LusternikSchnirelmann category of Ganea [6]. This invariant was introduced as an approximation for cat, the category of Lusternik and Schnirelmann. In its turn cat $(M)+1$, [9], gives a lower bound for the minimal number of critical points of a smooth function on a smooth, closed manifold $M$; cat $X$ may be defined as the least natural number $n$ such that there are $n+1$ open subsets of $X$ whose union covers $X$, each of them being contractible to the base point in $X$. A description of Cat $X$, that does not involve the notion of cone-length, is the same as that of cat except that the subsets covering $X$ are required to be contractible in themselves. Obviously cat $X \leq$ Cat $X$. Ganea and Takens [11] proved also that Cat $X \leq$ cat $X+1$. For further reference let us mention here two alternative definitions for cat . The first one, due to Whitehead, asserts that

Received by the editors September 30, 1993.

1991 Mathematics Subject Classification. Primary 55P50; Secondary 55P62. 
cat $X \leq n$ iff the diagonal $X \rightarrow X^{n+1}$ factors, up to homotopy, through the inclusion of the fat wedge $T^{n+1} X \hookrightarrow X^{n+1}$. The second one, given by Ganea, states that cat $X \leq n$ iff the $n$th Milnor fibration:

$$
E_{n} \Omega X \rightarrow B_{n} \Omega X \rightarrow X
$$

appearing in the classifying construction for $\Omega X$ admits a section up to homotopy.

2. $\mathscr{F}_{i}=$ the class of suspensions of order $i$. Denote $\mathrm{Cl}=\mathrm{Cl}_{\mathscr{F}}$ in this case. This notion was introduced in [2] where it is shown that $\mathrm{Cl} \leq \mathrm{cat}+1$.

3. $\mathscr{F}_{i}=$ wedges of spheres. Let $\mathrm{Cl}_{S}$ be the resulting invariant. It was introduced (in the rational context) by Lemaire and Sigrist in [8]. Notice that, if we take $\mathscr{F}_{i}=$ wedges of spheres of dimension at least $i$, the invariant will not change.

In the context of the three examples above we have $\mathrm{Cl}_{S} \geq \mathrm{Cl} \geq \mathrm{Cat} \geq$ cat . In fact, $\mathrm{Cl}_{S}$ and Cat are the extreme examples of cone-length.

Let $\mathscr{S}_{0}$ be the category of rational simply-connected spaces of finite type. The definition 1 may be used to define similar notions in $\mathscr{S}_{0}$ with some obvious modifications: we start the cofibration sequences with $i \geq 1$, define $X_{1}$ to be of the homotopy type of the rationalization of a simply-connected suspension and require the spaces in $\mathscr{F}_{i}$ to be simply-connected $(i \geq 1)$. We preserve the same notations as in $\mathscr{S}$.

Remark. In $\mathscr{S}_{0}$ we have $\mathrm{Cl}_{S}=\mathrm{Cl}$ because rational suspensions are wedges of spheres.

In the above-mentioned paper, Lemaire and Sigrist conjectured $\mathrm{Cl}_{S}=$ cat in $\mathscr{S}_{0}$. This problem is the origin of the present work. An immediate consequence of our main result is that, in this rational setting, $\mathrm{Cl}_{S}=\mathrm{Cat}$.

The key to even formulate our result, and moreover to prove it, is a good understanding of the dictionary geometry $\leftrightarrow$ algebra specific to rational homotopy. It allows translations of different geometric notions (in particular, the various types of cone-length) in the category of rational, connected, graded, differential Lie algebras of finite type, $\mathscr{D} \mathscr{S} \mathscr{L}$, and in the category of rational, commutative, graded, differentiable, simply-connected algebras of finite type, $\mathscr{D G \mathscr { A }}$, (we refer to [7], [12] for the basics of rational homotopy).

To state our theorem we need a few more preliminaries.

Let $h-\mathscr{S}$ be the category of homotopy types of objects in $\mathscr{S}$ with morphisms given by homotopy classes of maps.

Each notion of cone-length induces a certain filtration on $h-\mathscr{S}$. Denote by $\mathscr{C} \mathscr{L}_{\mathscr{F}}$ the filtration induced by $\mathrm{Cl}_{\mathscr{F}}:(\mathscr{C} \mathscr{L} \mathscr{F})_{n}$ consists of the homotopy types of objects $X$ in $\mathscr{S}$ such that $\mathrm{Cl}_{\mathscr{F}} X \leq n$ and homotopy classes of maps which admit a level preserving representative in the sense that if $\mathrm{Cl}_{\mathscr{g}} X \leq n$, $\mathrm{Cl}_{\mathscr{F}} X^{\prime} \leq n$, then $f: X \rightarrow X^{\prime}$ is level preserving if they are objects of $\mathscr{S}: Z_{i}$, $Z_{i}^{\prime} \in \mathscr{F}_{i}, X_{i}, X_{i}^{\prime}$ and morphisms $g_{i}, f_{i}$ where $1 \leq i<n$ such that the following diagrams are commutative:

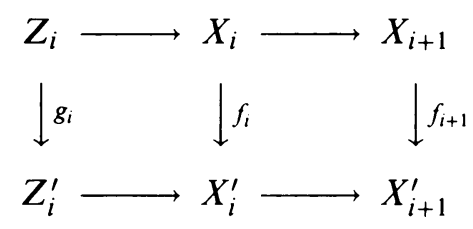


the rows are cofibration sequences, $X_{0}=X_{0}^{\prime}=*, f_{0}=\mathrm{id}, X_{n} \simeq X, X_{n}^{\prime} \simeq X^{\prime}$, $f_{n} \simeq f$.

Let $\mathscr{C} \mathscr{A} \mathscr{T}$ and $\mathscr{C} \mathscr{L}_{S}$ be the filtrations given by the previous construction using respectively Cat and $\mathrm{Cl}_{S}$. We will use the same notations for the corresponding two filtrations obtained in $\mathscr{S}_{0}$ (here, of course, in the definition of a level preserving map we start with $f_{1}$ ) and for the filtrations obtained by translating everything in $\mathscr{D} \mathscr{E} \mathscr{L}$ language.

One of the main ingredients of our analysis of the rational cone-length is the approach introduced by Felix and Halperin in the study of the rational L. S. category.

Definition 2. Let $A \in \mathscr{D} \mathscr{A}$. The nil-length of $A$, nil $A$, is the least natural number $n$ (or $\infty$ ) for which there is a quasi-isomorphism $A \rightarrow B$, with $B \in$ $\mathscr{D} \mathscr{A}$ and $\bar{B}^{n+1}=0(\bar{B}$ is the augmentation ideal of $B)$. For $X \in \mathscr{S}_{0}$, $\operatorname{nil} X=\operatorname{nil}\left(\mathscr{M}_{X}\right)$ where $\mathscr{M}_{X}$ is Sullivan's minimal model of $X$.

We might think about nil $X$ as the homotopic nilpotency of the algebra of P.L. forms on $X$.

In [4] Felix and Halperin give the following description for the L.S. category. If $X \in \mathscr{S}_{0}$, cat $X \leq n$ iff $X$ is a retract of some $Y \in \mathscr{S}_{0}$ such that nil $Y \leq n$. They also ask about a geometric interpretation for nil. As we shall see, another immediate consequence of our theorem is that $\mathrm{Cat}=$ nil .

Remark. It was shown in [2] that in the previous definition $B$ does not have to be simply-connected, or finite type. If we require only that it is an augmented algebra we will still get the same invariant. In the same paper it was also shown that nil $=\mathrm{Cl}$.

The nil-length induces a filtration on $h-\mathscr{D} \mathscr{G} A$ that we will denote by $\mathscr{N} \mathscr{L}: \mathscr{N G L}_{n}$ consists of the homotopy types of algebras $A$ with nil $A \leq n$ together with homotopy classes of morphisms that admit representatives $g: A \rightarrow$ $A^{\prime}$ with $\bar{A}^{n+1}=0,{\overline{A^{\prime}}}^{n+1}=0$.

Recall now the existence of a (classical) pair of functors $\mathscr{L}_{*}: \mathscr{D G \mathscr { A }} \leftrightarrow \mathscr{D} \mathscr{\mathscr { L }}$ : $\mathscr{C}^{*},[10]$, which are homotopy adjoint.

Theorem 1.1. The functor $L:(h-\mathscr{D} \mathscr{\mathscr { A }}) \rightarrow(h-\mathscr{D} \mathscr{\mathscr { L }})$ induced by $\mathscr{L}_{*}$ preserves filtrations with respect to $\mathscr{N} \mathscr{S L}$ and $\mathscr{C} \mathscr{L} S$.

The functor $C:(h-\mathscr{D} \mathscr{L} \mathscr{L}) \rightarrow(h-\mathscr{D} \mathscr{S} \mathscr{A})$ induced by $\mathscr{C}^{*}$ preserves filtrations with respect to $\mathscr{C} A \mathcal{S}$ and $\mathscr{N} \mathscr{L}$.

Corollary 2. In $h-\mathscr{D} \mathscr{S} \mathscr{L}$ we have $\mathscr{C} \mathscr{L}_{S}=\mathscr{C} \mathscr{A} \mathscr{T}$.

Remarks. 1. This implies not only that $\mathrm{Cl}_{S}=\mathrm{Cat}$ (everything is rational) but also any homotopy class of maps that admits a level preserving representative in the sense of Cat admits also a level preserving representative in the sense of $\mathrm{Cl}_{S}$; such a homotopy class of maps can be represented inside the $\mathscr{D} \mathscr{E} \mathscr{A}$ 's by a "short" model: a morphism between two "short" algebras.

2. The main cases when the Lemaire-Sigrist conjecture is known to hold are those of spaces which are formal or coformal or with cat $\leq 2$, [5]; in particular $\mathrm{Cat}=\mathrm{Cl}_{S}$ was known to hold only in these cases.

The next two sections are devoted to the proof of the theorem. Below we would like to make a few comments on some closely related problems: 
1. In the geometric (nonrational) context a natural problem is to distinguish among the different notions of cone-length. An approach to this question, along some quite classical lines, is to consider a cohomology theory $h^{*}(-)$ together with some natural transformations $\psi^{i}: h^{*} \rightarrow h^{*}, i \in I$; suppose that the $\psi^{i}$ 's vanish on $\Sigma \mathscr{F}$ (in the sense that $\psi^{i}(u)=0$ for $u \in h^{*}(\Sigma X), X \in \mathscr{F}, i \in I$ ). If for some space $Y$ there are $i_{1}, i_{2}, \ldots, i_{n} \in I$ such that $\psi^{i_{1}} \psi^{i_{2}} \ldots \psi^{i_{n}}(u) \neq 0$ for some $u \in h^{*}(Y)$, then $\mathrm{Cl}_{\mathscr{F}} Y \geq n+1$. For example ordinary $\mathbf{Z} / 2$ cohomology together with the Steenrod squares may be used to show that $\mathrm{Cl}_{S}-\mathrm{Cl}$ can reach arbitrarily large values.

Two basic questions remain open:

2. Rationally are Cat and cat different?

3. Geometrically are $\mathrm{Cl}$ and Cat different? In this context it is worth mentioning that Ganea, [6], and more recently Clapp and Puppe, [1], have given some criteria that insure the equality of Cat and cat. The most efficient such condition (given in [1]) is: Cat $X=$ cat $X$ if $X$ is $p$-connected, $p \geq 1$, and $\operatorname{dim} X \leq(2$ cat $X+1)(p+1)-3$.

The author would like to thank Joe Neisendorfer for his many helpful suggestions and for his advice, Jon Moore for his sustained interest in these problems, Steve Halperin for many useful discussions and for suggesting to us the statement (and the usefulness) of Lemma 2.2, and Jean-Paul Doeraene for some helpful remarks.

\section{2. $L$ PRESERVES FILTRATIONS}

We want to show here that for any differential graded algebra morphism: $f: \mathscr{A} \rightarrow \mathscr{B}$, with $\mathscr{A}, \mathscr{B} \in \mathscr{D} \mathscr{A}, \overline{\mathscr{A}}^{n+1}=\overline{\mathscr{B}}^{n+1}=0$ the induced morphism $\mathscr{L}_{*}(f): \mathscr{L}_{*}(\mathscr{B}) \rightarrow \mathscr{L}_{*}(A)$ fits into a homotopy commutative diagram:

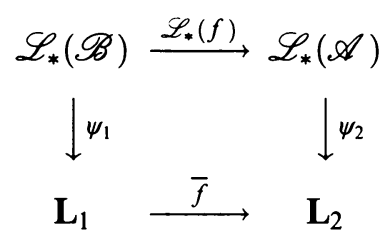

where $\psi_{1}, \psi_{2}$ are quasi-isomorphisms, $\mathbf{L}_{1}=\left(\mathbf{L}\left(X_{1}\right), d_{1}\right), \mathbf{L}_{2}=\left(\mathbf{L}\left(X_{2}\right), d_{2}\right)$ $(\mathbf{L}(X)$ being the free Lie algebra on $X)$ and $\mathbf{L}_{1}, \mathbf{L}_{2}, \bar{f}$ satisfy two conditions:

1. There are filtrations of $X_{j}(j=0,1), 0=F_{0} X_{j} \subset \cdots \subset F_{n} X_{j}=X_{j}$ with $d_{j}\left(F_{i+1} X_{j}\right) \subset \mathbf{L}\left(F_{i} X_{j}\right)$.

Having these filtrations in place let $F_{i} Z_{j}$ be a complement of $F_{i} X_{j}$ in $F_{i+1} X_{j}$. Let $\mathbf{L}_{i j}^{\prime}=\left(\mathbf{L}\left(F_{i} Z_{j} \oplus s^{-1} F_{i} Z_{j}\right), d_{i j}\right)$ where $s^{-1}$ is the desuspension and $d_{i j}(x)=s^{-1} x$, for $x \in F_{i} Z_{j}, d_{i j}(y)=0$ for $y \in s^{-1} F_{i} Z_{j}$. Let $\mathbf{L}_{i j}=$ $\left(\mathbf{L}\left(s^{-1} F_{i} Z_{j}\right), 0\right)$. There are unique morphisms $u_{i j}, v_{i j}$ :

$$
\mathbf{L}_{i j} \stackrel{u_{i j}}{\longrightarrow}\left(\mathbf{L}\left(F_{i} X_{j}\right), d_{j}\right) * \mathbf{L}_{i j}^{\prime} \stackrel{v_{i j}}{\longrightarrow}\left(\mathbf{L}\left(F_{i+1} X_{j}\right), d_{j}\right)
$$

such that $v_{i j}$ restricts to inclusion on $F_{i} X_{j}$ and on $F_{i} Z_{j}, u_{i j}(y)=y-d(s y)$ for $y \in \mathbf{L}_{i j}$. The existence of these morphisms is equivalent, geometrically, to the fact that the space represented by $\left(\mathbf{L}\left(F_{i+1} X_{j}\right), d_{j}\right)$ is the cofibre of a map from a wedge of spheres, represented by $\mathbf{L}_{i j}$, into the space represented by $\left(\mathbf{L}\left(F_{i} X_{j}\right), d_{j}\right)$. The morphism $\bar{f}$ has to satisfy: 
2. There are morphisms $f_{i}, g_{i}, 0 \leq i<n, f_{n}=\bar{f}$ such that the following diagrams commute:

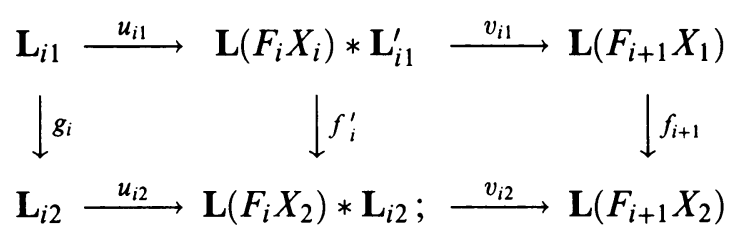

where $f_{i}^{\prime}=f_{i} * g_{i} * s g_{i}$ (notice that $\mathbf{L}_{i j}^{\prime}$ is isomorphic, as Lie algebra, with $\left.\mathbf{L}_{i j} * s \mathbf{L}_{i j}\right)$. This condition means, in Lie algebra language, that $\bar{f}$ is "level preserving".

It is easy to see that to verify 2 , it is enough to check that:

$2^{\prime}$. The maps $f_{i}$ as above exist and are compatible with the inclusions $\mathbf{L}\left(F_{i} X_{j}\right) \hookrightarrow \mathbf{L}\left(F_{i+1} X_{j}\right)$ and, for some choice of $F_{i} Z_{j}$, the image of the restriction of $f_{i+1}$ to $F_{i} Z_{1}$ is contained in $F_{i} Z_{2}$.

We will prove our claim by induction over $n$.

There is nothing to prove for $n=0$. The induction step is provided by the next result.

Proposition 2.1. let $f: \mathscr{A} \rightarrow \mathscr{B}$ be a $\mathscr{D} \mathscr{G} \mathscr{A}$-morphism with $\overline{\mathscr{A}}^{n+1}=\overline{\mathscr{B}}^{n+1}=0$. There is a $\mathscr{D} \mathscr{S} \mathscr{L}$-morphism $\bar{f}$ making the diagram below homotopy commutative:

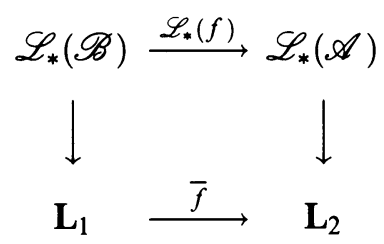

such that $\mathbf{L}_{1}, \mathbf{L}_{2}$ are free as Lie algebras, the vertical arrows are quasi-isomorphisms, $\mathbf{L}_{1}=\left(\mathbf{L}_{1}^{\prime} * \mathbf{L}_{1}^{\prime \prime}, d_{1}\right), \mathbf{L}_{2}=\left(\mathbf{L}_{2}^{\prime} * \mathbf{L}_{2}^{\prime \prime}, d_{2}\right)$, if $\mathbf{L}_{i}=\mathbf{L}\left(U_{i}\right)$, then $d_{i}\left(U_{i}\right) \subset \mathbf{L}_{i}^{\prime}$ for $i=1,2, \bar{f}\left(\mathbf{L}_{1}^{\prime}\right) \subset \mathbf{L}_{2}^{\prime}, \bar{f}\left(\mathbf{L}_{1}^{\prime \prime}\right) \subset \mathbf{L}_{2}^{\prime \prime}$ and:

$$
\left(\mathbf{L}_{1}^{\prime}, d_{1}\right) \simeq \mathscr{L}_{*}\left(\mathscr{B} \mid \overline{\mathscr{B}}^{n}\right), \quad\left(\mathbf{L}_{2}^{\prime}, d_{2}\right) \simeq \mathscr{L}_{*}\left(\mathscr{A} / \overline{\mathscr{A}}^{n}\right) .
$$

Remark. This proposition implies that, in the given circumstances, $\mathscr{A}$ and $\mathscr{B}$ represent spaces which are cofibres of some maps from wedges of spheres to the spaces represented, respectively, by $\mathscr{A} / \overline{\mathscr{A}}^{n}$ and $\mathscr{B} / \overline{\mathscr{B}}^{n}$ and $f$ represents a map that can be made level preserving with respect to these cofibration sequences.

The proposition follows from the next two lemmas.

Lemma 2.2. Let $f: \mathscr{A} \rightarrow \mathscr{B}$ be a $\mathscr{D} \mathscr{G}$-morphism and let $I \subset \mathscr{A}, J \subset \mathscr{B}$ be two ideals such that $f^{-1}(J)=I$, the restriction of $f$ to $I$ is injective and $\overline{\mathscr{A}} \cdot I=0, \mathscr{\mathscr { B }} \cdot J=0$. There is a $\mathscr{D} \mathscr{G} \mathscr{L}$-morphism $\bar{f}$ with all the properties in the statement of the proposition with the exception of $(*)$ which is replaced by $\left(\mathbf{L}_{1}^{\prime}, d_{1}\right) \simeq \mathscr{L}_{*}(\mathscr{B} / J),\left(\mathbf{L}_{2}^{\prime}, d_{2}\right) \simeq \mathscr{L}_{*}(\mathscr{A} / I)$.

Remark. The proof of this lemma is based on the ideas of [2, $\S 3$, Proposition 3.3]. This statement and its utility in the present context was pointed out to us by Steve Halperin. 
Proof. Recall the functor $\mathscr{L}_{*}: \mathscr{D G \mathscr { A }} \rightarrow \mathscr{D \mathscr { L }}$. Let $\mathscr{C D \mathscr { C } C}$ be the category of cocommutative, differential, graded, simply-connected coalgebras of finite type, then $\mathscr{L}_{*}=\mathscr{L}$ ○\# where \#: $\mathscr{D} \mathscr{G} \mathscr{A} \rightarrow \mathscr{C} \mathscr{D} \mathscr{C}$ is a dualization functor and $\mathscr{L}: \mathscr{C} \mathscr{D} \mathscr{C} \rightarrow \mathscr{D} \mathscr{L} \mathscr{L}$ verifies: $\mathscr{L}(C, \delta)=\left(\mathbf{L}\left(s^{-1} C\right), d^{\prime}+d^{\prime \prime}\right), d^{\prime}, d^{\prime \prime}$ induced by $d^{\prime}\left(s^{-1} x\right)=-s^{-1}(\delta x), d^{\prime \prime}\left(s^{-1} x\right)=(-1 / 2) \Sigma(-1)^{\left|a_{i}\right|}\left[s^{-1} a_{i}, s^{-1} b_{i}\right]$ for $\bar{\Delta} x=\sum a_{i} \otimes b_{i}, x \in C, \Delta$ being the coproduct in $C$ (we will use $\#(A)=$ $\left.A^{*}\right)$.

Let us return to our lemma. We have vector space isomorphisms $\mathscr{A} \simeq$ $(\mathscr{A} / I) \oplus I, \mathscr{B} \simeq(\mathscr{B} / J) \oplus J$. Choose vector space bases for $\mathscr{A}$ and $\mathscr{B}$ which respect these splittings. Dualize with respect to these bases. We get $\mathscr{L}_{*}(\mathscr{B})=\left(\mathbf{L}_{1}^{\prime} * \mathbf{L}_{1}^{\prime \prime}, d_{1}\right), \mathscr{L}_{*}(\mathscr{A})=\left(\mathbf{L}_{2}^{\prime} * \mathbf{L}_{2}^{\prime \prime}, d_{2}\right)$ where $\mathbf{L}_{1}^{\prime} \simeq \mathbf{L}\left(s^{-1}(\mathscr{B} / J)^{*}\right)$, $\mathbf{L}_{2}^{\prime} \simeq \mathbf{L}\left(s^{-1}(\mathscr{A} / I)^{*}\right), \quad \mathbf{L}_{1}^{\prime \prime} \simeq \mathbf{L}\left(s^{-1}(J)^{*}\right), \quad \mathbf{L}_{2}^{\prime \prime} \simeq \mathbf{L}\left(s^{-1}(I)^{*}\right)$. It is immediate to check that $\mathbf{L}_{1}^{\prime}$ and $\mathbf{L}_{2}^{\prime}$ are closed to $d_{1}$ respectively to $d_{2}$ and that we have isomorphisms: $\left(\mathbf{L}_{1}^{\prime}, d_{1}\right) \simeq \mathscr{L}_{*}(\mathscr{B} / J),\left(\mathbf{L}_{2}^{\prime}, d_{2}\right) \simeq \mathscr{L}_{*}(\mathscr{A} / I)$. Moreover, because $f^{-1}(I)=J$ the map $\mathscr{L}_{*}(f)$ respects the splittings on $\mathscr{L}_{*}(\mathscr{B})$ and $\mathscr{L}_{*}(\mathscr{A})$. The ideal $J$ can be written, when viewed as a differential graded vector space, as a sum $J=H_{1} \oplus U_{1}$ such that each $h \in H_{1}$, is a cocycle and $U_{1}$ is acyclic. A similar splitting exists on $I$ and, because $f$ is monomorphic on $I$, we can choose $I=H_{2} \oplus U_{2}$ such that $f^{-1}\left(H_{1}\right) \subset H_{2}$. As a consequence of this we get splittings $\mathbf{L}_{i}^{\prime \prime}=l_{i}^{\prime} * l_{i}^{\prime \prime}$ where $l_{i}^{\prime}=\mathbf{L}\left(s^{-1} H_{i}^{*}\right), l_{i}^{\prime \prime}=\mathbf{L}\left(s^{-1} U_{i}^{*}\right)$, $(i=1,2)$. Remark that $\mathscr{L}_{*}(f)\left(l_{1}^{\prime}\right) \subset l_{2}^{\prime}$. Let us denote $\mathbf{L}_{i}=\mathbf{L}_{i}^{\prime} * l_{i}^{\prime}$.

We have Lie algebra maps: $\mathbf{L}_{1} \stackrel{j_{1}}{\rightarrow} \mathscr{L}_{*}(\mathscr{B}) \stackrel{r_{1}}{\rightarrow} \mathbf{L}_{1}, \mathbf{L}_{2} \stackrel{j_{2}}{\rightarrow} \mathscr{L}_{*}(\mathscr{A}) \stackrel{r_{2}}{\rightarrow} \mathbf{L}_{2}$ with $r_{i} \circ j_{i}=\mathrm{id}, j_{i}$ being obvious inclusions, $r_{i}$ projections. Moreover we have the following commutative diagram:

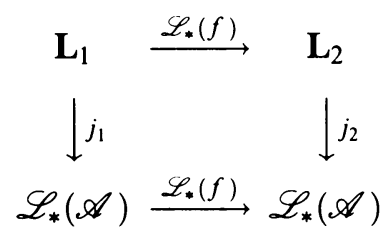

Here the top arrow is the restriction of $\mathscr{L}_{*}(f)$ to $\mathbf{L}_{1}$. Let $d_{i}=d_{i}^{\prime}+d_{i}^{\prime \prime}$ be the standard splitting of the differential in $\mathscr{L}_{*}(\mathscr{B})$ for $i=1$ and in $\mathscr{L}_{*}(\mathscr{A})$ for $i=2$. If $x$ is a generator of $l_{i}^{\prime}$ then $d_{i}^{\prime} x=0$; because $\overline{\mathscr{B}} \cdot J=0$ and $\overline{\mathscr{A}} \cdot I=0$ it is easy to see that $d_{i}^{\prime \prime} x \in \mathbf{L}_{i}^{\prime}$. This means that $d_{i}$ admits a restriction to $\mathbf{L}_{i}$. With respect to this induced differential $r_{i}$ becomes a differential map. To conclude the proof let us just notice that both $r_{i}$ and $j_{i}$ are quasi-isomorphisms. Indeed $l_{i}^{\prime \prime}$ is $d_{i}^{\prime}$ acyclic, hence it is $d_{i}$ acyclic.

We would now like to apply the previous lemma to the case of $I=\overline{\mathscr{A}}^{n}$, $J=\overline{\mathscr{B}}^{n}$. To do that we have to show that the conditions in the statement of the lemma may be satisfied.

Lemma 2.3. Let $f: \mathscr{A} \rightarrow \mathscr{B}$ be a $\mathscr{D \mathscr { G }}$-morphism with $\overline{\mathscr{A}}^{n+1}=\overline{\mathscr{B}}^{m+1}=0$, $m \leq n$. It is possible to factor $f: \mathscr{A} \stackrel{f_{1}}{\rightarrow} \mathscr{A} \otimes_{d} E \stackrel{f_{2}}{\rightarrow} \mathscr{B}, f_{1}$ being the inclusion $x \rightarrow x \otimes 1, f_{2}$ is a quasi-isomorphism, ${\overline{\left(\mathscr{A} \otimes_{d} E\right)}}^{n+1}=0$ (clearly $E$ is not free as an algebra).

Proof. Let $\mathscr{A}_{0} \stackrel{f^{\prime}}{\rightarrow} \mathscr{A}$ be a minimal model of $\mathscr{A}$. It is a standard fact [3] that the product $\mu: \mathscr{A}_{0} \otimes \mathscr{A}_{0} \rightarrow \mathscr{A}_{0}$ admits an extension $m:\left(\mathscr{A}_{0} \otimes \mathscr{A}\right) \otimes_{d} \mathscr{A}^{\prime} \rightarrow \mathscr{A}_{0}$ where if $\mathscr{A}_{0}=(\Lambda Z, d), \mathscr{A}^{\prime}=\left(\Lambda s^{-1} Z, 0\right)$ and the twisted tensor product 
$\left(\mathscr{A}_{0} \otimes \mathscr{A}_{0}\right) \otimes_{d} \mathscr{A}^{\prime}$ corresponds, geometrically, to the fibration $\Omega X \rightarrow X \stackrel{\Delta}{\rightarrow} X \times X$ where $X$ is a space represented by $\mathscr{A}_{0}$ and $\Delta$ is the diagonal (notice that $\mathscr{A}^{\prime}$ represents $\Omega X$ ). It is easy to check that the same construction works for $\mathscr{A}$. That means that we get a map $t:(\mathscr{A} \otimes \mathscr{A}) \otimes_{d} \mathscr{A}^{\prime} \rightarrow \mathscr{A}$ extending the multiplication $\mathscr{A} \otimes \mathscr{A} \rightarrow \mathscr{A} ; t$ (as $m$ before) is easily seen to be a quasiisomorphism.

Now $f$ turns $\mathscr{B}$ into an $\mathscr{A}$-module. Using this structure we get $j_{1}=$ $t \otimes_{\mathscr{A}} \mathscr{B}:(\mathscr{A} \otimes \mathscr{B}) \otimes_{d} \mathscr{A}^{\prime} \rightarrow \mathscr{B}$ which will still be a quasi-isomorphism. We have obtained a factorization of $f$ :

$$
\mathscr{A} \stackrel{i_{1}}{\longrightarrow}(\mathscr{A} \otimes \mathscr{B}) \otimes_{d} \mathscr{A}^{\prime} \stackrel{j_{1}}{\longrightarrow} \mathscr{B},
$$

$i_{1}$ is injective, $j_{1}$ is a quasi-isomorphism. Let $T_{1}=(\mathscr{A} \otimes \mathscr{B}) \otimes_{d} \mathscr{A}^{\prime}$. We also have a factorization of the identity $1_{\mathscr{B}}$ :

$$
\mathscr{B} \stackrel{h_{1}}{\longrightarrow}(\mathscr{A} \otimes \mathscr{B}) \otimes_{d} \mathscr{A}^{\prime} \stackrel{j_{1}}{\longrightarrow} \mathscr{B}
$$

where $h_{1}(x)=1 \otimes x \otimes 1$.

Suppose we have constructed $T_{n}$ and factorizations of $f$ and $1_{\mathscr{B}}$ respectively: $\mathscr{A} \stackrel{i_{n}}{\rightarrow} T_{n} \stackrel{j_{n}}{\rightarrow} \mathscr{B}, \mathscr{B} \stackrel{h_{n}}{\rightarrow} T_{n} \stackrel{j_{n}}{\rightarrow} \mathscr{B}$. Build the next step by remarking, first, that $j_{n}$ factors $j_{n}: T_{n} \rightarrow T_{n}{\overline{\left(T_{n}\right)}}^{n+1} \stackrel{i_{n}}{\rightarrow} \mathscr{B}$ and construct $T_{n+1}=$ $\left(T_{n}{\overline{\left(T_{n}\right)}}^{n+1}\right) \otimes_{d} \mathscr{F}_{n}, \mathscr{F}_{n}$ free, together with morphisms $h_{n+1}: \mathscr{B} \rightarrow T_{n+1}$ and $j_{n+1}: T_{n+1} \rightarrow \mathscr{B}$ such that both are quasi-isomorphisms and $j_{n+1} \circ h_{n+1}=1_{\mathscr{B}}$. We can now define $i_{n+1}: \mathscr{A} \rightarrow T_{n+1}$ by the composition $\mathscr{A} \stackrel{i_{n}}{\rightarrow} T_{n} \rightarrow T_{n+1}$. Details on the construction of $T_{n+1}$ together with the fact that passing to the limit (with respect to $n$ ) gives a splitting of $1_{\mathscr{B}}: \mathscr{B} \stackrel{i}{\rightarrow} T \stackrel{j}{\rightarrow} \mathscr{B}$ with $h, j$ quasi-isomorphisms, $T$ of finite type, simply-connected and $\bar{T}^{n+1}=0$ can be found in $[2, \S 3]$. At the limit we get also a map $\mathscr{A} \stackrel{f_{1}}{\rightarrow} T$ and $\mathscr{A} \stackrel{f_{1}}{\rightarrow} T \stackrel{j}{\rightarrow} \mathscr{B}$ is our factorization of $f$.

Finally, to end the proof of the proposition, apply the above lemma to replace $f: \mathscr{A} \rightarrow \mathscr{B}$ by $f_{1}: \mathscr{A} \rightarrow \mathscr{A} \otimes_{d} E=\mathscr{B}^{\prime}$ which is a monomorphism and satisfies $f_{1}^{-1}\left(\overline{\mathscr{B}}^{\prime}\right)=\overline{\mathscr{A}}^{n}$. Now use Lemma 2.2 with $I=\overline{\mathscr{A}}^{n}, J=\overline{\mathscr{B}}^{\prime}$.

\section{3. $C$ PRESERVES FILTRATIONS}

We recall that, if not mentioned otherwise, we are working inside the category of simply-connected, finite type rational spaces. It is immediate that our claim on $C$ reduces to the next proposition.

Proposition 3.1. Suppose the following diagram is commutative:

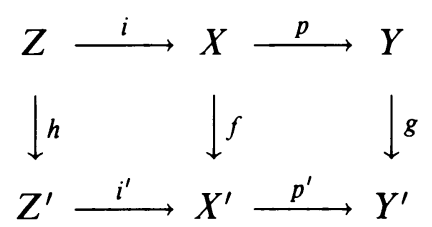

where the rows are cofibration sequences and $f$ admits a representative: $\bar{f}: \mathscr{A}^{\prime}$ $\rightarrow \mathscr{A} ; \mathscr{A}, \mathscr{A}^{\prime} \in \mathscr{D G \mathscr { A }}, \overline{\mathscr{A}}^{n+1}=\overline{\mathscr{A}}^{n+1}=0, \mathscr{A}^{\prime}$ representing $X^{\prime}$ and $\mathscr{A}$ representing $X$. Then $g$ admits a representative $\bar{g}: \mathscr{B}^{\prime} \rightarrow \mathscr{B} ; \mathscr{B}, \mathscr{B}^{\prime} \in$ $\mathscr{D} \mathscr{\mathscr { A }}, \overline{\mathscr{B}}^{\prime+2}=\overline{\mathscr{B}}^{n+2}=0, \mathscr{B}^{\prime}$ representing $Y^{\prime}$ and $\mathscr{B}$ representing $Y$. 
Proof. Consider the following homotopy commutative diagram:

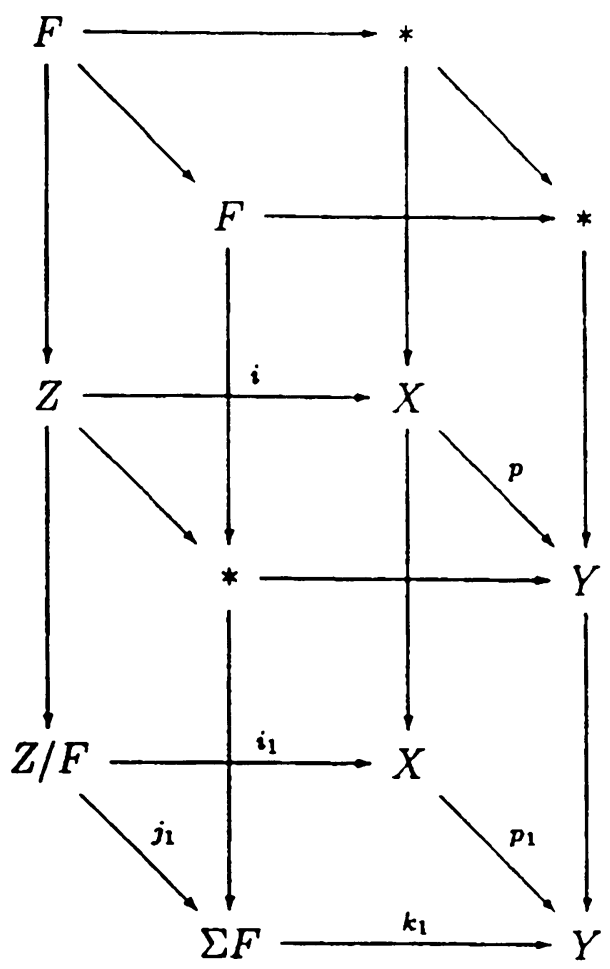

Here $F$ is the homotopy fibre of $i$ (and it might not be simply-connected, however the fibration is clearly nilpotent), the vertical sequences are cofibrations and, as the top and middle square are push-out diagrams, so is the bottom square. Clearly a similar diagram exists in the ' case. We denote by $F^{\prime}$ the homotopy fibre of $i^{\prime}$. We obtain a map of push-out squares:

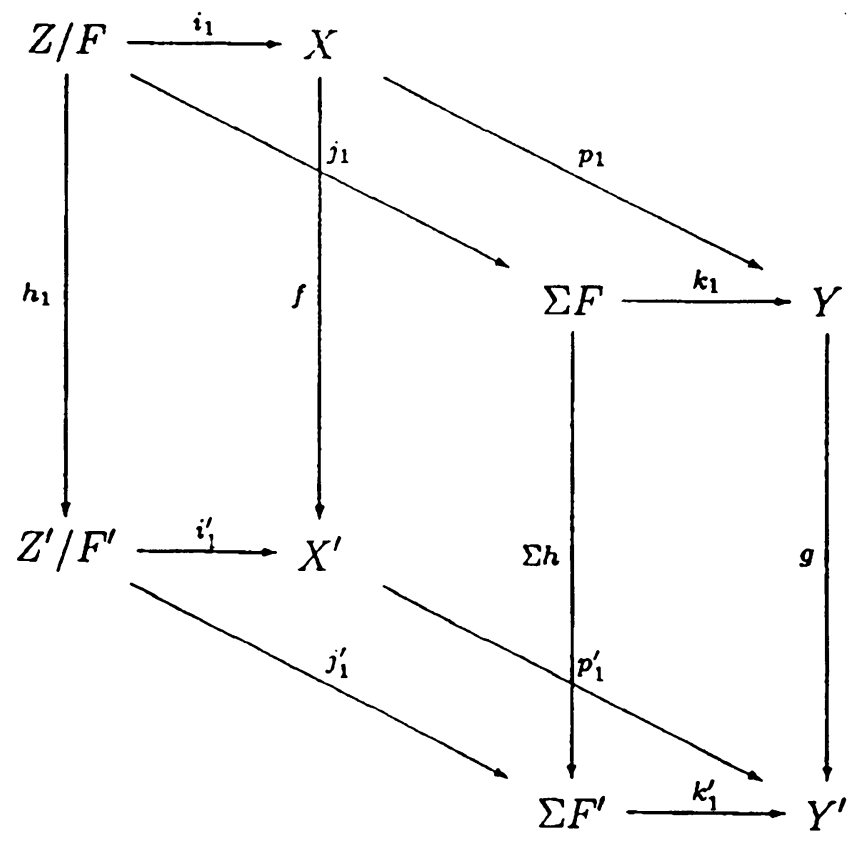


Recall that at the center of Sullivan's point of view on rational homotopy there is the functor "piecewise linear forms" $A_{P L}(-)$. If we apply this functor to the previous diagram we get two short exact sequences of augmented algebras:

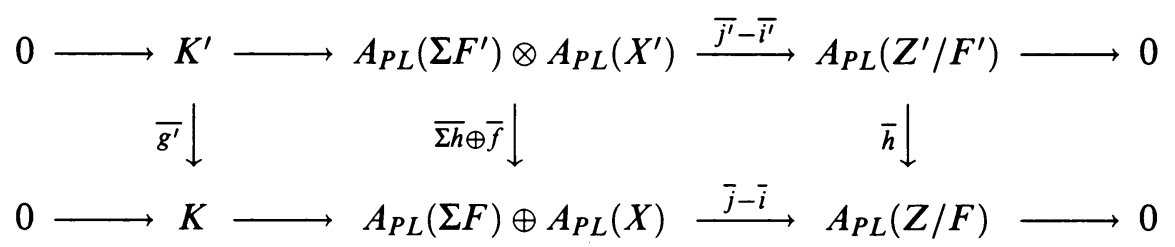

Here $\bar{j}=A_{P L}\left(J_{1}\right), \overline{j^{\prime}}=A_{P L}\left(j_{1}^{\prime}\right), \overline{i^{\prime}}=A_{P L}\left(i^{\prime}\right), \bar{i}=A_{P L}(i), \overline{\Sigma h}=A_{P L}(\Sigma h)$, $\bar{f}=A_{P L}(f), \bar{h}=A_{P L}\left(h_{1}\right)$. By the elementary properties of the piecewise linear forms we get that $\overline{g^{\prime}}$ is a representative for $g$.

Remark. The maps $\bar{j}-\bar{i}$ and $\overline{j^{\prime}}-\overline{i^{\prime}}$ are not algebra maps. However $K$ and $K^{\prime}$ are clearly subalgebras of the respective algebras.

Next we plan to represent the previous exact sequences in terms of algebras as short as possible (in the sense that the nilpotency degree of the augmentation ideal of such an algebra should be as small as possible).

If we view the map $i: Z \rightarrow X$ as a fibration, by a standard technique of rational homotopy [7] we may represent $i$ by an inclusion $i_{2}: \mathscr{A} \rightarrow \mathscr{A} \otimes_{d} \mathscr{F}$ where $\mathscr{A}$ represents $X$ (and appears in the statement of the proposition), $\mathscr{F}$ is the Sullivan model of $F$. Moreover the projection $\mathscr{A} \otimes_{d} \mathscr{F} \rightarrow \mathscr{F}$ represents the inclusion of the fibre $F \hookrightarrow Z$. Hence the map $i_{1}: Z / F \rightarrow X$ will be represented by $i_{3}: \mathscr{A} \rightarrow \mathbf{Q} \oplus\left(\overline{\mathscr{A}} \otimes_{d} \mathscr{F}\right)=T ; i_{3}$ is uniquely defined by the property that when composed with the inclusion $T \hookrightarrow \mathscr{A} \otimes_{d} \mathscr{F}$ it gives $i_{2}$. Everything we did till now can be done in the ${ }^{\prime}$ case and even in a functorial way, hence we get a square:

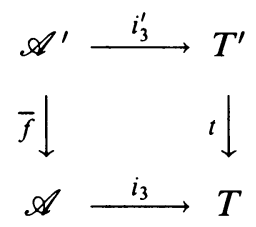

which represents the square $(*)$ :

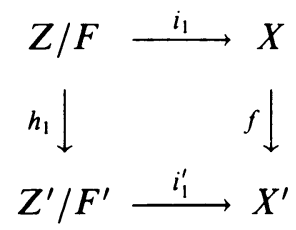

Remark that $\bar{T}^{n+1}={\overline{T^{\prime}}}^{n+1}=0$. We would like to show now that $i_{3}$ and $i_{3}^{\prime}$ can be perturbed as to become surjective. 


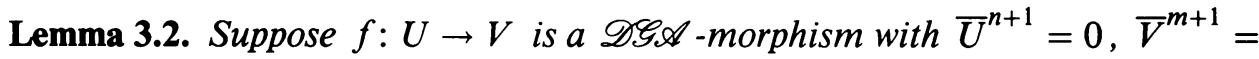
$0, m \leq n$. Then it is possible to factor $f$ in $U \stackrel{f^{\prime}}{\rightarrow} U^{\prime} \stackrel{f^{\prime \prime}}{\rightarrow} V$ with $f^{\prime \prime}$ surjective and $f^{\prime}$ a quasi-isomorphism, ${\overline{U^{\prime}}}^{n+1}=0$.

Proof. By the basic properties of rational homotopy we can first factor $f$ in $U \stackrel{f_{1}}{\rightarrow} U_{1} \stackrel{f_{2}}{\rightarrow} V$ where $U_{1}=U \otimes_{d} L, L$ being an acyclic, free, differential, graded algebra, $f_{1}$ the obvious inclusion (in particular it is a quasi-isomorphism) and $f_{2}$ is surjective.

Let $J={\overline{U_{1}}}^{n+1}$. As $m \leq n, f_{2}$ factors $U_{1} \rightarrow U_{1} / J \rightarrow V$. It is obvious that our claim is proved if we show that $J$ is acyclic. Let $\left\{\left(E_{r}\left(U_{1}\right), d_{r}\right)\right\}$ be the spectral sequence obtained by filtering $U_{1}$ by the powers of the augmentation ideal. To show that $J$ is acyclic it is enough to notice that $E_{1}^{\geq n+1, *}\left(U_{1}\right)=0$. But this follows from the short exact sequence below:

$$
0 \rightarrow \bar{U} \otimes_{d} L \rightarrow U_{1} \rightarrow L \rightarrow 0
$$

Because $E_{1}^{\geq n+1, *}\left(\bar{U} \otimes_{d} L\right)=0, E_{1}(L)=\mathbf{Q}$ where the involved spectral sequences are also induced by filtrations by the powers of the augmentation ideals.

Applying the previous lemma first to $i_{3}$ and then to $i_{3}^{\prime}$ we will get a new square:

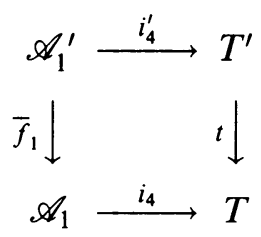

representing the square $(*)$ and such that both $i_{4}$ and $i_{4}^{\prime}$ are surjective and $\overline{\mathscr{A}}_{1}^{n+1}=\overline{\mathscr{A}}_{1}^{n+1}=0$.

The next step is to represent in a good way the diagram $(* *)$ :

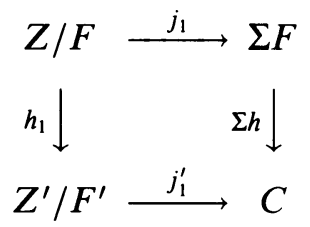

Standard rational homotopy theory allows us to represent this square by:

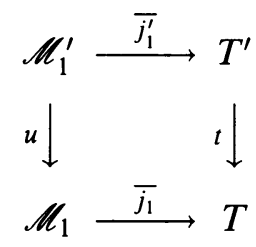

Where $\mathscr{M}_{1}, \mathscr{M}_{1}^{\prime}$ are free models of $\Sigma F, \Sigma F^{\prime}$ respectively, both $\overline{j_{1}}, \overline{j_{1}^{\prime}}$ are surjective and $u$ represents $h_{1}$. Moreover, because $\Sigma F, \Sigma F^{\prime}$ are both coformal spaces, we may assume that the differentials in $\mathscr{M}_{1}$ and $\mathscr{M}_{1}^{\prime}$ are at most quadratic. 


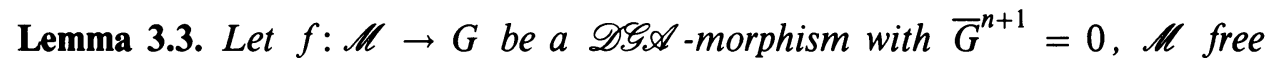
with a differential at most quadratic. If nil $\mathscr{M}=m$ then there is a factorization of $f: \mathscr{M} \stackrel{f^{\prime}}{\rightarrow} \mathscr{M}^{\prime} \stackrel{f^{\prime \prime}}{\rightarrow} G$ such that $f^{\prime}$ is a quasi-isomorphism and $\overline{\mathscr{M}}^{p+1}=0$ for any $p \geq \max \{m, n+1\}$.

Proof. Let $\left\{\left(E_{r}(\mathscr{M}), d_{r}\right)\right\}$ be the spectral sequence induced by the filtration by the powers of the augmentation ideal of $\mathscr{M}$. Because the differential of $\mathscr{M}$ is at most quadratic it follows that $d_{r}=0$ for $r \geq 2$. Also, because nil $\mathscr{M}=m$ we get $E_{2}^{>m, *}(\mathscr{M})=0$. In particular if $p \geq \max \{m, n+1\}$ we get $E_{2}^{>p, *}(\mathscr{M})=0$. Let $I=\overline{\mathscr{M}}^{p+1}$. It is clear that $f$ factors: $\mathscr{M} \rightarrow \mathscr{M} / I \stackrel{f_{1}}{\rightarrow} G$. The ideal $I$ is not acyclic in general, however, $E_{2}^{>p, *}(\mathscr{M})=0$ implies that we can find a minimal set of elements $U=\left\{v_{i}\right\}$ such that $v_{i} \in \overline{\mathscr{M}}^{p}, v_{i}$ survives to $E_{1}$ and $d_{1}\left(\left[v_{i}\right]\right)$ spans the image of $d_{1}: E_{1}^{p, *} \rightarrow E_{1}^{p+1, *}$. Define now $J=I \oplus \mathbf{Q}\{U\}$. This is an acyclic ideal and, because $p \geq n+1$ we notice that $f_{1}(U)=0$. Hence we get the wanted factorization of $f: \mathscr{M} \rightarrow \mathscr{M} / J \rightarrow G$.

Returning to the proposition, the spaces $\Sigma F$ and $\Sigma F^{\prime}$ are also formal, thus we have surjective quasi-isomorphisms: $\mathscr{M}_{1} \stackrel{\phi}{\rightarrow} H=H^{*}(\Sigma F), \mathscr{M}_{1}^{\prime} \stackrel{\phi^{\prime}}{\rightarrow} H^{\prime}=$ $H^{*}\left(\Sigma F^{\prime}\right)$. Hence nil $\mathscr{M}_{1}^{\prime}=$ nil $\mathscr{M}_{1}=1$. By using the lemma above we can represent the square $(* *)$ by:

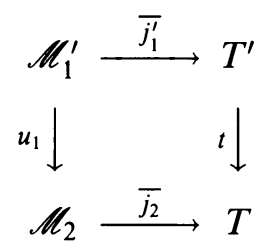

with $\overline{\mathscr{M}}_{2}^{n+2}=0$.

We cannot apply directly the same construction to $\mathscr{M}_{1}^{\prime}$ because the construction of $U$ in the proof of the lemma involves some choices that are not functorial. However the lemma gives us an ideal $J^{\prime} \subset \mathscr{M}_{1}^{\prime}$ such that $\overline{j_{1}^{\prime}}$ factors: $\mathscr{M}_{1}^{\prime} \stackrel{\psi}{\rightarrow} \mathscr{M}_{1}^{\prime} / J^{\prime} \stackrel{s}{\rightarrow} T^{\prime},{\overline{\left(\mathscr{M}_{1}^{\prime} / J^{\prime}\right)}}^{n+2}=0$ with $\psi$ a quasi-isomorphism. Now $\phi$ factors through $\mathscr{M}_{2}: \mathscr{M}_{1} \rightarrow \mathscr{M}_{1} / J=\mathscr{M}_{2} \stackrel{\phi_{1}}{\rightarrow} H$. Consider the composition $u_{2}: \mathscr{M}_{1}^{\prime} \stackrel{u_{1}}{\rightarrow} \mathscr{M}_{2} \stackrel{\phi_{1}}{\rightarrow} H$. Clearly, $u_{2}$ is null on $J^{\prime}$ hence it factors through $\psi$.

We get the following commutative diagram $(* * *)$ :

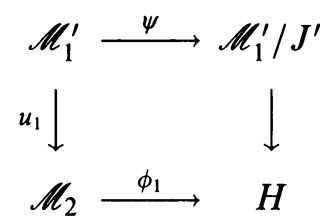

where $\psi$ and $\phi_{1}$ are surjective quasi-isomorphisms and $\overline{\mathscr{M}}_{2}^{n+2}={\overline{\left(\mathscr{K}_{1}^{\prime} / J^{\prime}\right.}}^{n+2}=$ 0 .

Lemma 3.4. Suppose we have the following commutative diagram (of $\mathscr{D} \mathscr{G}$ 's):

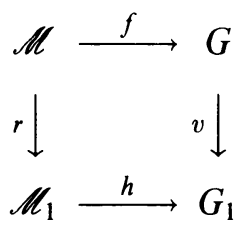


with $r, v$ quasi-isomorphisms, $v$ surjective and $\overline{\mathscr{M}}_{1}^{n+1}={\overline{G_{1}}}^{n+1}=\bar{G}^{n+1}=0$, then there exists a $\mathscr{D} \mathscr{\mathscr { A }}, \mathscr{M}^{\prime}$ together with maps $e: \mathscr{M} \rightarrow \mathscr{M}^{\prime}, k: \mathscr{M}^{\prime} \rightarrow G$, $l: \mathscr{M}^{\prime} \rightarrow \mathscr{M}_{1}$ such that $l \circ e=r, k \circ e=f, e$ a quasi-isomorphism and $\overline{\mathscr{M}}^{n+1}=0$.

Proof. The construction we will use is very similar to that of Lemma 2.2 (and Lemma 3.7 of [2]).

It is clear that both $f$ and $r$ factor through $\mathscr{M} / \overline{\mathscr{M}}^{n+1}$. Let $T_{0}=\mathscr{M}$, $f_{0}=f, r_{0}=r, e_{0}=\mathrm{id} ;$ we have the commutative diagram (0):

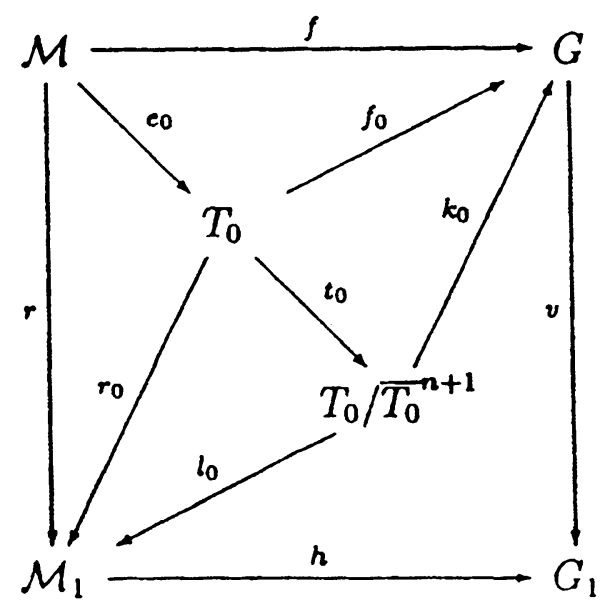

Suppose that, by induction, we have constructed a diagram (i) with the property that is the same as (0) but all indexes 0 are replaced by $i, e_{i}$ is a quasiisomorphism and $t_{i} \circ e_{i}$ is at least $(i+1)$-connected. Define $T_{i+1}=T_{i} / \bar{T}_{i}^{n+1} \otimes_{d} Z_{i}$ (with $Z_{i}$ free) by successively killing the kernel of $H^{*}\left(l_{i}\right)$. Define $e_{i+1}$ to be the composition $\mathscr{M} \rightarrow T_{i} \rightarrow T_{i} / \bar{T}_{i}^{n+1} \rightarrow T_{i+1} ; t_{i+1}$ is the obvious projection. It is easy to check that $t_{i+1} \circ e_{i+1}$ is at least $i+2$-connected. The map $r_{i+1}$ appears by the construction of $T_{i+1}$. Let us show that we can also construct $f_{i+1}$. We define it to be $k_{i}$ on $T_{i} / \bar{T}_{i}^{n+1}$. We have to show that we can extend it also over $Z_{i}$ (see Lemma 3.7 in [2]). The initial step is given by taking $\alpha \in T_{i} / \bar{T}_{i}^{n+1}$ such that $[\alpha] \in \operatorname{Ker} H^{*}\left(l_{i}\right)$ and suppose $\beta \in Z_{i}$ verifies $d \beta=\alpha$. Then $h \circ l_{i}(\alpha)=d \beta^{\prime}$. Also $\beta^{\prime}=v\left(\beta^{\prime \prime}\right)$ because $v$ is surjective. We have $v \circ k_{i}(\alpha)=h \circ l_{i}(\alpha)$, hence $d \beta^{\prime \prime}-k_{i}(\alpha) \in \operatorname{Ker}(v)$. But as $v$ is a quasi-isomorphism, and $d\left(d \beta^{\prime \prime}-k_{i}(\alpha)\right)=0$ there is a $\gamma \in G$ such that $d \gamma=d \beta^{\prime \prime}-k_{i}(\alpha)$. Define $f_{i+1}(\beta)=\beta^{\prime \prime}-\gamma$. The same type of argument may be applied at later stages in the construction of $Z_{i}$. Passing to the limit we get $\mathscr{K}^{\prime}$ as the limit of the $T_{i}$ 's, $l$ as that of the $l_{i}$ 's and $k$ as that of the $k_{i}$ 's.

Returning now to the diagram $(* * *)$, by applying the previous lemma we get an algebra $\mathscr{K}_{2}^{\prime}$ and maps making the next diagram commutative: 


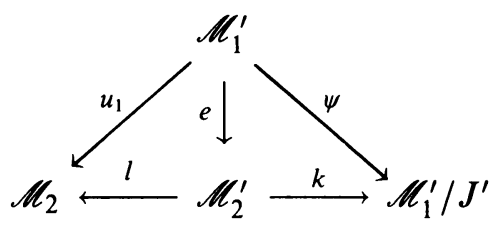

with $\overline{\mathscr{M}}_{2}^{n+2}=\overline{\mathscr{M}}_{2}^{n+2}=0, e, \psi, k$ quasi-isomorphisms, $k$ surjective. Making use of this we can represent the square $(* *)$ by:

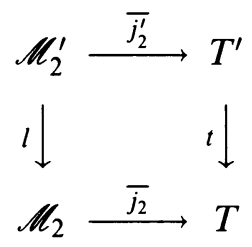

where $\overline{j_{2}^{\prime}}=s \circ k$. Recall that $\overline{\mathscr{M}}_{2}^{n+2}=\overline{\mathscr{M}}_{2}^{\prime n+2}=\bar{T}^{n+1}={\overline{T^{\prime}}}^{n+1}=0$. We may also suppose $\overline{j_{2}}, \overline{j_{2}^{\prime}}$ surjective (by Lemma 3.1 ). Putting things together we get the following map of short exact sequences of augmented algebras:

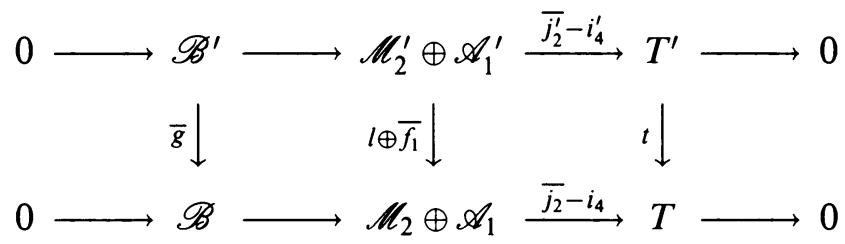

The map $\bar{g}$ represents $g$ and $\overline{\mathscr{B}}^{n+2}=\overline{\mathscr{B}}^{n+2}=0$.

Remark. In [2] it is proved that nil $=\mathrm{Cl}$ by showing that a map $\Sigma F \rightarrow X$ can be represented in terms of short algebras. In fact the ideal kind of proof for nil $\leq$ cat would be along the same lines: perturb a map $Z \rightarrow Y$ without changing its cofibre such that it becomes representable in terms of short algebras.

\section{REFERENCES}

1. M. Clapp and D. Puppe, Invariants of the Lusternik-Schnirelmann type and the topology of critical sets, Trans. Amer. Math. Soc. 298 (1986), 603-620.

2. O. Cornea, Cone-length and Lusternik-Schnirelmann category, Topology (to appear).

3. Y. Félix, La dichotomie elliptique-hyperbolique en homotopie rationnelle, Astérisque 176, Soc. Math. de France, 1989.

4. Y. Félix and S. Halperin, Rational L.S. category and its applications, Trans. Amer. Math. Soc. 273 (1982), 1-37.

5. Y. Félix and J. C. Thomas, Sur la structure des espaces de L.S. catégorie deux, Illinois J. Math. 30 (1986), 574-593.

6. T. Ganea, Lusternik-Schnirelmann category and strong category, Illinois J. Math. 11 (1967), 417-427.

7. S. Halperin, Lectures on minimal models, Mem. Soc. Math. France 9-10 (1983).

8. J.-M. Lemaire and F. Sigrist, Sur les invariants d'homotopie rationnelle liés à la L.S. catégorie, Comment. Math. Helv. 56 (1981), 103-122.

9. L. Ljusternik and L. Schnirelmann, Méthodes topologiques dans les problèmes variationnels, Hermann, Paris, 1934. 
10. D. Quillen, Rational homotopy the, Annals of Math. 90 (1985), 205-295.

11. F. Takens, The Lusternik-Schnirelmann categories of a product space, Comput. Math. 22 (1970), 175-180.

12. D. Tanré, Homotopie rationnelle: Modéles de Chen, Quillen, Sullivan, Lecture Notes in Math., vol. 1025, Springer-Verlag, Berlin and New York, 1983.

Department of Mathematics, University of Rochester, Rochester, New York 14627

Current address: Department of Mathematics, University of Toronto, Toronto, Canada

E-mail address: cornea@math.toronto.edu 